British Heart fournal, 1977, 39, 471-476

\title{
Rapid mobilisation after acute myocardial infarction First step in rehabilitation and secondary prevention
}

\author{
P. E. THORNLEY AND R. W. D. TURNER
}

From the Chest Unit, City Hospital, Edinburgh, and the Western General Hospital, Edinburgh

(1)

Rapid mobilisation after acute myocardial infarction as the first step in a planned programme of rehabilitation and secondary prevention was studied in a group of 142 men under 65 years of age admitted to a coronary care unit.

Patients without complications were mobilised after 2 days' bed rest, but the programme was flexible and those with complications were mobilised less rapidly. Fifteen patients died before mobilisation could be started. In 127 who survived long enough to be mobilised, 74 (58\%) spent 2 to 4 days in bed, 42 (33\%) 5 to 10 days, and $11(9 \%)$ more than 10 days. The mean period of bed rest was 5.4 days.

Rapid mobilisation eliminated the need for physiotherapy and led to early discharge from hospital with more economic use of hospital beds. In 125 patients who were discharged, 99 (79\%) had 16 days or less (5 to 16) in hospital. The mean period of hospital stay was 15.5 days.

A programme of progressive rehabilitation with advice on secondary prevention, which was started in hospital, was continued at a convalescent hospital with graded exercises supervised by physiotherapists. This allayed anxiety and led to a more confident return home. A community nurse visited the home to reinforce advice given in hospital to the patient and his wife and extend primary prevention to the family. Review was undertaken at a special clinic to ensure the safety of the programme and to give further reassurance and advice about early return to work, exercise, and secondary prevention. Cardiac neurosis was thereby virtually prevented. In 105 eligible for employment, 46 per cent had returned to work within 12 weeks and 86 per cent within 24 weeks, of whom 90 per cent returned to their former employment.

In 127 patients who had been mobilised, $3(2.5 \%)$ died within 6 weeks, and a further $8(6.5 \%)$ had various nonfatal cardiac complications, but none of the 74 mobilised rapidly after 2 to 4 days died within this period. Only one was readmitted within 4 weeks.

In view of this and other reported experiences, it is clear that rapid mobilisation and early discharge after myocardial infarction should now be standard practice and there is no need of further evidence of its safety.

As recently as 1969 , a 3-week period of bed rest after myocardial infarction was accepted practice in Britain (Lancet, 1969). Since then there has been a trend toward faster mobilisation and earlier discharge from hospital, but practice still varies widely (Heasman and Carstairs, 1971) even between physicians in the same hospital (Duke, 1971). In 1973 patients in a Glasgow hospital had 7 days in bed and 3 weeks in hospital (Medical Division, Royal Infirmary, Glasgow, 1973), in Stoke on Trent 1 day in bed and 8 days in hospital (Tucker et al., 1973), while Datey in Bombay allowed only 25 per cent of survivors of acute infarction to walk 30 yards after 2 weeks (Datey et al., 1973).

Received for publication 16 August 1976
It is unusual for British physicians to supervise later rehabilitation or secondary prevention after discharge from hospital (Royal College of Physicians, 1975). In 1971, in answer to a questionnaire, 113 members of the British Cardiac Society stated that they were in favour of some form of rehabilitation service, but 80 had no facilities (Groden et al., 1971).

This variation in practice, which generally does not include full rehabilitation, led us to examine the results of our method of rapid mobilisation linked to a planned programme of continued rehabilitation and secondary prevention. The method was simple and did not require elaborate facilities. Other advantages included a short hospital stay, confident 
return home, early return to work, and prevention of cardiac neurosis.

Hurst in a recent Editorial (Hurst, 1975) pointed out the importance of considering all patients, with and without complications and including deaths, in any analysis of early ambulation and that many who do have complications, unless severe, benefit from early mobilisation. In this study all have been included.

\section{Patients}

One-hundred-and-forty-two men under the age of 65 were admitted under the care of one of the physicians in charge of the coronary care unit (CCU) of the Western General Hospital in Edinburgh. Myocardial infarction was diagnosed when there was a convincing history in the preceding 48 hours together with either (a) an acute rise to at least twice the normal upper limit of serum aspartate aminotransferase or serum lactic dehydrogenase, or (b) electrocardiographic changes of 'certain' or 'very probable' myocardial infarction by WHO criteria 1959 (World Health Organisation, 1959).

The mean age was 54.9 (36 to 64 ) years.

The delay between onset of symptoms and admission was less than $\mathbf{4 8}$ hours in all cases (mean 10.4 hours) and less than 8 hours in two-thirds of them.

The position of the infarct was anterior in 59 $(42 \%)$, inferior in $34(24 \%)$, posterior in $3(2 \%)$, combined sited in $26(18 \%)$, and unclassified in 20 $(14 \%)$ because of bundle-branch block or incompletely evolved electrocardiographic abnormalities before death.

Seventy-two $(51 \%)$ patients had a past history of coronary heart disease, $45(30.5 \%)$ had stable angina, and $13(9 \%)$ had had unstable angina in the preceding month.

\section{Method}

Admission was to a Coronary Care Unit with conventional facilities for monitoring and treatment of emergencies. Patients were under the care of the staff on duty while in the unit, but were subsequently discharged to a general ward under one of the physicians in rotation according to the day of admission. Consequently, each physician received patients admitted on different days of the week, resulting in effective randomisation. All patients in this series were under the charge of one physician (R.W.D.T.).

Initially all were treated in bed but allowed to wash, shave, and feed themselves and, unless too ill, to use a bedside commode. Warfarin was given for prophylaxis of venous thrombosis, unless contraindicated, but withdrawn as soon as the patient was ambulant. Patients free from cardiac pain, shock, hypotension, abnormal praecordial pulsation, significant cardiac failure or arrhythmia were allowed to sit in a chair after two days in bed. On the third day a few steps around the bed were allowed and after this they were permitted to walk freely about the ward until fully mobile. The programme of mobilisation was flexible and adjusted to individual need so that some with complications were mobilised more slowly.

During this time their medical condition was explained in simple terms. Wives were seen by a dietitian and given advice on a suitable calorie, low-saturated fat, and low-cholesterol diet with partial substitution by polyunsaturated fat and restriction of sugar. Smokers were advised dogmatically to stop. Each patient was given an explanatory booklet on coronary risk factors.

In addition, all patients were seen by a community nurse who acted as a link between hospital, home, and family doctor. She made further inquiries about the domestic and working situations and later made a home visit to reinforce advice given in hospital, answer questions, and extend advice to the family by way of primary prevention.

Usually, after 10 to 14 days, a two-week period in a convalescent hospital was recommended. Active rehabilitation with exercise to improve muscle tone and joint mobility, followed by progressive walking, was supervised by physiotherapists. Most were told they would probably be taking more exercise within a few months than for many years past.

On discharge from the convalescent hospital, review was undertaken at a special outpatient clinic, again after 3 months (or sooner if indicated), and thereafter at longer intervals. At each visit, after inquiring, a brief physical examination was made and blood taken for measurement of plasma lipids. An electrocardiogram and $x$-ray examination were carried out if indicated.

The purpose of continued supervision was to allay anxiety, ensure the safety of progressive activity and to encourage adherence to the secondary prevention regimen and early return to work.

\section{Results}

Information was complete on 140 of 142 patients; unfortunately, 2 were not given clinic appointments in error, and later they could not be traced.

MISCELIANEOUS FACTORS

Bed rest

Fifteen patients died while still confined to bed. 
This left 127 to be mobilised; $74(58 \%)$ had 2 to 4 days in bed, $27(21 \%) 5$ to 7 days, $15(12 \%) 8$ to 10 days, and $11(9 \%)$ more than 10 days. The range was 2 to 33 days, with a mean of 5.4 days. Fifty-one were considered unfit for mobilisation before the 5 th day because of arrhythmia (28), defect of conduction (9), cardiac pain (8), recurrent infarction $(1)$, and cardiac failure (7).

\section{Duration of hospital stay}

Two patients died in hospital after mobilisation. Of the 125 discharged $86(69 \%)$ were in hospital for 14 days or less. The range of hospital stay was 5 to 99 days, with a mean of $15 \cdot 1$ days (Table 1 ). The number of days in hospital was partly determined by the availability of a convalescent bed and, had one been available on the day required, $100(86 \%)$ would have been transferred on the 12 to 14 th day.

\section{Convalescent hospital stay}

One-hundred-and-four patients stayed in the convalescent hospital for a mean of 12.1 days. Six discharged themselves early and 3 had to be readmitted to the Coronary Care Unit. Twenty-one did not go to the convalescent hospital for a variety of reasons, which included patient refusal and long distance from home, making it inconvenient for relatives to visit them.

\section{Time off work}

Seven patients died within 24 weeks before returning to work, and 11 were retired or redundant before admission to hospital. Information was incomplete in two. In the remaining 105 eligible for return to work, $48(46 \%)$ returned within 12 weeks, $73(70 \%)$ within 16 weeks, and $90(86 \%)$ within 24 weeks (Table 2).

In the $15(14 \%)$ who had not returned to work within 24 weeks, 7 had angina or dyspnoea and 5 had previously been employed in heavy manual labour and were advised to change to less strenuous

Table 1 Length of stay in hospital

\begin{tabular}{llllll}
\hline Days in hospital & $5-12$ & $13-14$ & $15-16$ & $>16$ & Total \\
\hline No. of patients & 49 & 37 & 13 & 26 & 125 \\
& $(39 \%)$ & $(30 \%)$ & $(10 \%)$ & $(21 \%)$ & $(100 \%)$ \\
\hline
\end{tabular}

Table 2 Length of stay off work

\begin{tabular}{lllllll}
\hline Time off work (wk) & $<8$ & $9-12$ & $13-16$ & $17-24$ & $>24$ & Total \\
\hline No. of patients & 17 & 31 & 25 & 17 & 15 & 105 \\
& $(16 \%)$ & $(30 \%)$ & $(24 \%)$ & $(16 \%)$ & $(14 \%)$ & $(100 \%)$ \\
\hline
\end{tabular}

work but were unwilling to take alternative employment with less pay. There was no satisfactory reason for prolonged time off work in the remaining patient.

\section{Change of occupation}

Of the 90 patients returning to work within 24 weeks, $81(90 \%)$ resumed their former employment. The reason given for a change in occupation included the employer's offer of different work in 6 , and advice from the hospital physician in 1. Two patients themselves decided that alternative work would be more suitable.

\section{MORTALITY}

Altogether 25 patients died within 24 weeks of the acute attack $(17.6 \%)$. This included 15 who died within 16 days of admission during bed rest and, being unrelated to the mobilisation programme, are not considered further. The 10 deaths that occurred within 24 weeks in the 127 patients mobilised are analysed below.

Deaths within 6 weeks after mobilisation (Table 3)

Three patients died within 6 weeks and each had had 5 to 10 days initial bed rest. Two had prolonged cardiac failure from the time of admission until death, indicating severe infarction. Neither had evidence of cardiac rupture at necropsy. A third was well until sudden death at home in the 5th week. None of the $\mathbf{7 4}$ mobilised after 2 to 4 days' bed rest died within 6 weeks. It is clear that too rapid mobilisation did not lead to an excess of deaths in the early postinfarction period.

Deaths from 6 to 24 weeks after mobilisation (Table 3) Seven patients died within 6 to 24 weeks. Three had been mobilised slowly after more than 10 days' bed rest on account of severe infarction and early complications. Each had persistent cardiac failure until death, and one had pulmonary embolism.

The other 4 patients had been mobilised rapidly after 2 to 4 days' bed rest. One had ventricular fibrillation, in the 7th week, 2 died suddenly at home in the 10th and 12th weeks, and 1 died of recurrent infarction and cardiac failure in the 15th week. It is

Table 3 Mortality within 24 weeks in 127 patients mobilised

\begin{tabular}{|c|c|c|c|}
\hline \multicolumn{2}{|c|}{ Rapidity of mobilisation } & \multirow{2}{*}{$\begin{array}{l}\text { Deaths within } \\
6 \text { wk }\end{array}$} & \multirow{2}{*}{$\begin{array}{l}\text { Deaths from } 6 \\
\text { to } 24 \text { wk }\end{array}$} \\
\hline No. of patients & Days bed rest & & \\
\hline $\begin{array}{l}74 \\
42 \\
11\end{array}$ & $\begin{array}{l}2-4 \\
5-10 \\
>10\end{array}$ & $\frac{-}{3}$ & $\frac{4}{3}$ \\
\hline
\end{tabular}


unlikely that these 7 deaths, occurring so long after infarction, were related to early mobilisation.

NON-FATAL CARDIAC COMPLICATIONS

Non-fatal cardiac complications within 6 weeks after mobilisation (Table 4)

Eight $(6.5 \%)$ of the 127 mobilised had 9 non-fatal cardiac complications within 6 weeks. (One patient had recurrent myocardial infarction in hospital and was later readmitted with cardiac pain.)

Non-fatal cardiac complications from 6 to 24 weeks after mobilisation (Table 5)

Six $(5 \%)$ of the 127 mobilised had 8 non-fatal cardiac complications from 6 to 24 weeks. (One patient was admitted 3 times with cardiac pain.) Some patients had more than one non-fatal cardiac complication and some patients who died had previously had non-fatal cardiac complications. After 6 weeks in the 127 mobilised, $116(91 \%)$ were uncomplicated, and after 24 weeks, $105(88 \%)$ were uncomplicated.

\section{READMISSIONS}

Altogether 12 patients were readmitted on 17 occasions within 24 weeks. Seven were readmitted within 6 weeks, but only one within 4 weeks. Thus early discharge did not result in early readmission.

\section{THROMBOEMBOLIC DISEASE}

Thromboembolic disease was diagnosed clinically and, where indicated, by chest $x$-ray and lung scanning. Each of the 7 patients with pulmonary

Table 4 Nonfatal cardiac complications within 6 weeks in 127 patients mobilised

\begin{tabular}{llll}
\hline Complication & \multicolumn{3}{l}{ Days of bed rest } \\
\cline { 2 - 4 } & $\begin{array}{l}2-4 \text { days } \\
(74 \text { patients })\end{array}$ & $\begin{array}{c}5-10 \text { days } \\
(42 \text { patients })\end{array}$ & $\begin{array}{l}10 \text { days } \\
(11 \text { patients })\end{array}$ \\
\hline Reinfarction definite & 2 & - & - \\
suspected & 3 & - & 1 \\
Cardiac failure & 1 & - & - \\
Ventricular fibrillation & - & 1 & - \\
Atrial fibrillation & - & 1 & - \\
\hline
\end{tabular}

Table 5 Nonfatal cardiac complication from 6 to 24 weeks in 127 patients mobilised

\begin{tabular}{llll}
\hline Complication & \multicolumn{4}{l}{ Days of bed rest } \\
\cline { 2 - 4 } & $\begin{array}{l}\text { 2-4 days } \\
\text { (74 patients) }\end{array}$ & $\begin{array}{c}5-10 \text { days } \\
\text { (42 patients) }\end{array}$ & $\begin{array}{l}\text { 10 days } \\
\text { (11 patients) }\end{array}$ \\
\hline Reinfarction definite & 2 & 1 & 1 \\
suspected & 1 & 2 & - \\
Cardiac failure & - & 1 & - \\
\hline
\end{tabular}

infarction had more than 8 days' bed rest. In these 7 patients, 4 developed pulmonary infarction during bed rest after the fourth day, and one later in hospital after mobilisation. The other 2 were readmitted because of pulmonary infarction and one patient died shortly after readmission.

A deep venous thrombosis was diagnosed clinically during bed rest in one patient who had pulmonary embolism.

Although thromboembolic disease occurred in patients who had prolonged bed rest of more than 8 days, these were patients who had severe infarction.

\section{VENTRICULAR ANEURYSM}

All patients were repeatedly examined by praecordial palpation. Cardiac screening was undertaken when ventricular aneurysm was suspected for any reason, but none was found.

\section{CARDIAC NEUROSIS}

Formal psychological testing was not undertaken. One patient developed depression and was transferred to a psychiatric hospital and another became depressed after 24 weeks. Overt cardiac neurosis was notably absent.

\section{Discussion}

\section{EARLY MOBILISATION AND DISCHARGE}

Mobilisation after uncomplicated myocardial infarction has been shown to be as safe after 2 days as after 9 days of bed rest (Hayes et al., 1974). Our study supports the safety of rapid mobilisation within a few days in uncomplicated cases. In the $\mathbf{7 4}$ patients mobilised after only 2 to 4 days in uncomplicated infarction or only transient complications, there were no deaths in 6 weeks and $4(5 \%)$ in 24 weeks. In a group of similarly uncomplicated patients mobilised after either 7 or 21 days' bed rest, Harpur et al. (1971) found mortality rates of 5 and 8 per cent, respectively, which were not statistically significantly different. Though strict comparison between different studies is not possible, we have no reason to suspect that a period of bed rest longer than 2 to 4 days is safer. It is, therefore, unnecessary.

There are fewer reports of mobilisation after complicated myocardial infarction. A recent controlled trial provided some evidence that mobilisation was not only safe, but safer after 6 to 8 days' bed rest, compared with 13 to 15 days' bed rest (Abraham et al., 1975). In our group there was a higher complication rate in the 53 patients mobilised after more than 4 days. In particular, there were 3 deaths in the 11 patients mobilised after 10 days out of a total of 
10 deaths in the 127 patients mobilised. However, the patients mobilised more slowly had more severe or prolonged complications.

An ideal trial should compare matched groups of patients with similar severity of infarction and similar complications undergoing several widely varying schedules. This would require large numbers and could not be done from a single centre. At the present time, without firm evidence about mobilisation after complicated myocardial infarction, commonsense dictates that any schedule should be flexible according to individual needs. We found that mobilisation was delayed beyond the fourth day in 42 per cent of survivors but only 9 per cent for longer than 10 days.

Datey et al. (1973) in Bombay, using selection criteria for ambulation which included absence of angina, cardiomegaly ( $x$-ray), ST shift of $2 \mathrm{~mm}$ or more on the electrocardiogram, abnormal blood pressure (systolic $<90 \mathrm{mmHg}$, diastolic $>100$ $\mathrm{mmHg}$ or significant orthostatic fall in mean pressure $>7 \mathrm{mmHg}$ ) or a resting heart rate $>100 / \mathrm{min}$, allowed only 25 per cent of survivors of myocardial infarction to walk 30 yards in 2 weeks. These criteria seem excessively strict and very likely to induce introspection and cardiac neurosis.

Advantages of early mobilisation are a shorter hospital stay for the patient and more economical use of hospital beds. In this sories, 69 per cent were discharged within 14 days.

Adgey (1969), in a group of patients spending less than 18 days in hospital after infarction, had no deaths or readmissions within 2 weeks of discharge. It was concluded that early discharge did not lead to early readmission or death from complications requiring resuscitation facilities. Similarly, we had only one readmission within 2 weeks of discharge and the earliest outpatient death occurred in the 5 th week after infarction. It could be argued that a prolonged hospital stay of 6 weeks would sometimes give a chance of resuscitation, but the results of late cardiac arrest are not good (Thompson and Sloman, 1971).

\section{REHABILITATION AND SECONDARY \\ PREVENTION}

It is unusual for British physicians to undertake more than a single review of patients discharged after myocardial infarction. We found that a period of convalescence with a graded exercise programme allayed anxiety and led to a more confident return home. Exercise was not medically supervised or monitored and there were no deaths during this period. A clearly explained regimen of graded exercises at home might serve equally well.

An intensive programme of secondary preven- tion was started after discharge from hospital. This also provided an opportunity for giving advice on aspects of rehabilitation other than secondary prevention, such as driving, sexual intercourse, and return to work. Patients were taught how to improve their own health and it was explained how a coronary attack could be the beginning of a more healthy way of life.

Return to work is the best form of rehabilitation. Nagle et al. (1971) found that only 50 per cent of a group of Birmingham car workers had returned to work within 16 weeks of myocardial infarction, in comparison with Royston (1972) who encouraged 90 per cent of patients back to work within 3 months with a policy of explanation and reassurance in hospital and at a review clinic. We agree that unequivocal advice can lead to a high rate of early return to work and found that 70 per cent had returned within 16 weeks and 79 per cent within 24 weeks.

Wishnie et al. (1971) found in a small group recovering from myocardial infarction that most felt anxious or depressed in the month after discharge, and about 30 per cent of these patients wished to return to hospital in the first week. Few had had advice about physical exercise and most were frustrated by inactivity. Wynn (1967) found that anxiety and depression accounted for disability in about 25 per cent of patients who remained unemployed after myocardial infarction. We did not undertake formal psychological assessment but were impressed by the lack of pyschiatric disturbance. We consider that cardiac neurosis can be avoided by clear explanation and a positive approach to rehabilitation.

At one time it was thought that early mobilisation might lead to ventricular aneurysm formation (Moyer et al., 1951). Our findings are in agreement with Harpur et al. (1971) who showed no difference in the incidence in patients mobilised after either 8 or 21 days and Groden et al. (1967) after 15 or 25 days.

It has been thought that bed rest predisposes to thromboembolism but Hayes et al. (1974) found no difference in the incidence of deep venous thrombosis detected by radioactive iodine labelled fibrinogen in patients mobilised rapidly after 2 days, or more slowly after 9 days. We have no evidence to show that rapid mobilisation reduced the incidence of thromboembolism because most of our patients also received anticoagulants. However, it is noteworthy that none of the uncomplicated patients who were mobilised after 2 to 4 days had clinical evidence of thromboembolism.

In 1972, Rose stated that the conservative physician who wishes to restrict his patients' recovery 
needs to prove his case. Our experience confirms the safety of rapid mobilisation and we consider that this practice should lead to early discharge, facilitate rehabilitation, and be the first stage in secondary prevention.

\section{References}

Abraham, A. S., Sever, Y., Weinstein, M., Dollberg, M., and Menczel, J. (1975). Value of early ambulation in patients with and without complications after acute myocardial infarction. New England fournal of Medicine, 292, 719-722.

Adgey, A. A. J. (1969). Prognosis after early discharge from hospital of patients with acute myocardial infarction. British Heart fournal, 31, 750-752.

Datey, K. K., Kapadia, N. M., Dalvi, C. P., and Pinto, I. J. (1973). Is very early ambulation safe in patients of acute myocardial infarction? Indian Heart fournal, 25, 303-307.

Duke, M. (1971). Bed rest in acute myocardial infarction. A study of physician practices. American Heart fournal, 82, 486-491.

Groden, B. M., Allison, A., and Shaw, G. B. (1967). Management of myocardial infarction-the effect of early mobilisation. Scottish Medical fournal, 12, 435-440.

Groden, B. M., Semple, T., and Shaw, G. B. (1971). Cardiac rehabilitation in Britain (1970). British Heart fournal, 33, 756-758.

Harpur, J. E., Conner, W. T., Hamilton, M., Kellett, R. J. Galbraith, H-J. B., Murray, J. J., Swallow, J. H., and Rose, G. A. (1971). Controlled trial of early mobilisation and discharge from hospital in uncomplicated myocardial infarction. Lancet, 2, 1331-1334.

Hayes, M. J., Morris, G. K., and Hampton, J. R. (1974). Comparison of mobilization after two and nine days in uncomplicated myocardial infarction. British Medical fournal, 3, 10-13.

Heasman, M. A., and Carstairs, V. (1971). Inpatient management: variations in some aspects of practice in Scotland. British Medical fournal, 1, 495-498.

Hurst, J. W. (1975). 'Ambulation' after myocardial infarction. New England fournal of Medicine, 292, 746-748.
Lancet (1969). Editorial. Early mobilisation after myocardial infarction. 1, 821.

Medical Division, Royal Infirmary, Glasgow (1973). Early mobilisation after uncomplicated myocardial infarction. Prospective study of 538 patients. Lancet, 2, 346-349.

Moyer, J. B., Hiller, G. I., and Detroit, M. (1951). Cardiac aneurysm: clinical and electrocardiographic analysis. American Heart fournal, 41, 340-358.

Nagle, R., Gangola, R., and Picton-Robinson, I. (1971). Factors influencing return to work after myocardial infarction. Lancet, 2, 454-456.

Rose, G. (1972). Early mobilization and discharge after myocardial infarction. Modern Concepts of Cardiovascular Disease, 41, 59-63.

Royal College of Physicians of London and British Cardiac Society (1975). Cardiac rehabilitation 1975. Fournal of the Royal College of Physicians of London, 9, 281-346.

Royston, G. R. (1972). Short stay hospital treatment and rapid rehabilitation of cases of myocardial infarction in a district hospital. British Heart fournal, 34, 526-532.

Thompson, P., and Sloman, G. (1971). Sudden death in hospital after discharge from coronary care unit. British Medical fournal, 4, 136-139.

Tucker, H. H., Carson, P. H. M., Bass, N. M., Sharratt, G. P., and Stock, J. P. P. (1973). Results of early mobilization and discharge after myocardial infarction. British Medical fournal, 1, 10-13.

Wishnie, H. A., Hackett, T. P., and Cassem, N. H. (1971). Psychological hazards of convalescence following myocardial infarction. Fournal of the American Medical Association, 215, 1292-1296.

World Health Organization (1959). The electrocardiogram in coronary heart disease. Technical Report Series, No. 168, pp. 25-28.

Wynn, A. (1967). Unwarranted emotional distress in men with ischaemic heart disease (IHD). Medical fournal of Australia, 2, 847-851.

Requests for reprints to Dr. R. W. D. Turner, University of Edinburgh, 21 Buccleuch Place, Edinburgh EH8 9LN. 